\title{
Density functional theory study of flat and stepped $\mathrm{NaCl}(001)$
}

\author{
Bo Li, ${ }^{1}$ Angelos Michaelides,,${ }^{1,2, *}$ and Matthias Scheffler ${ }^{1}$ \\ ${ }^{1}$ Fritz-Haber-Institut der Max-Planck-Gesellschaft, Faradayweg 4-6, D-14195 Berlin, Germany \\ ${ }^{2}$ London Centre for Nanotechnology and Department of Chemistry, University College London, London WC1E 6BT, United Kingdom
}

(Received 3 May 2007; revised manuscript received 21 June 2007; published 3 August 2007)

\begin{abstract}
The properties of bulk $\mathrm{NaCl}, \mathrm{NaCl}(001)$, and stepped $\mathrm{NaCl}(001)$ surfaces have been examined with density functional theory within the plane-wave pseudopotential approach. Aiming to remedy the lack of quantitative energetic and structural knowledge of such surfaces, we employ the local-density approximation and generalized gradient approximation of Perdew-Burke-Ernzerhof (PBE) exchange-correlation functionals as well as the PBE Wu-Cohen functional [Phys. Rev. B. 73, 235116 (2006)] to determine the surface energy and surface structure of $\mathrm{NaCl}(001)$. A range of $9-15 \mathrm{meV} / \AA^{2}$ is obtained for the surface energy of $\mathrm{NaCl}(001)$, and the surface is predicted to undergo only small relaxations of the top layer atoms, consistent with low-energy electron diffraction $I-V$ analyses. The isolated step formation energy of monoatomic (100)-like steps on $\mathrm{NaCl}(001)$ is estimated to be about $40-60 \mathrm{meV} / \AA$ and the interaction energy between adjacent steps is weak. Thermodynamics has been employed to determine the relative stabilities of stoichiometric (100)-like and nonstoichiometric (111)-like steps on $\mathrm{NaCl}(001)$, revealing that (100)-like steps are significantly more stable than (111)-like steps at all accessible values of the chlorine chemical potential.
\end{abstract}

DOI: 10.1103/PhysRevB.76.075401

PACS number(s): 73.20.At, 71.15.Mb, 73.61.Ng

\section{INTRODUCTION}

Common salt, $\mathrm{NaCl}$, plays a key role in environmental chemistry, biology, and several other scientific disciplines, as well as being a material of obvious importance to many aspects of daily life. Since all bodies interact with their surroundings through their surfaces, it is necessary to explore the structure and properties of $\mathrm{NaCl}$ surfaces if an understanding of the role salt and salt particles play in the above disciplines is to be arrived at. On the basis that one must first understand the properties of clean surfaces before one can understand their interaction with atoms and molecules, the current paper focuses exclusively on flat and stepped $\mathrm{NaCl}(001)$.

Many qualitative features of the surfaces of $\mathrm{NaCl}$ are well understood. ${ }^{1,2}$ It is known, for example, from the cubic equilibrium shape of $\mathrm{NaCl}$ crystals, that the low-energy surface of $\mathrm{NaCl}$ is the electrically neutral and stoichiometric (001) surface. It is further understood that the $\mathrm{Na}$ and $\mathrm{Cl}$ atoms at such surfaces will undergo only small relaxations from their bulk truncated positions. ${ }^{3}$ It is recognized that the steps, which invariably form at such surfaces, are stoichiometric and nonpolar and of importance to many physical and chemical properties of $\mathrm{NaCl}^{4-7}$ However, quantitative insight into the properties of $\mathrm{NaCl}$ surfaces is, to a large extent, lacking. This is mainly because many of the popular surface science probes, which have been so successfully exploited to probe the surfaces of metals, such as scanning tunneling microscopy, low-energy electron diffraction (LEED), and photoelectron spectroscopies, cannot easily be applied in a nondestructive manner to the surfaces of wide-band-gap ionic materials, such as $\mathrm{NaCl}$. The conventional application of these electron-based probes to insulators can lead to surface charging, electron-stimulated dissociation of the alkali halides, and alkali enrichment of the selvedge, all of which hampers characterization of the surface. ${ }^{8}$

From experiment there are two main approaches to remedy or at least mitigate the destructive nature of the electron- based probes at insulating surfaces. One is to use a probe with a low electron current. With LEED, for example, this means the deployment of systems operating with very low primary currents in the picoampere or low nanoampere range. The second approach is to investigate the properties of thin (nanometer) films of insulators supported on metallic substrates, rather than attempting to investigate pure single crystals of the ionic materials themselves. For $\mathrm{NaCl}(001)$, both techniques have been applied, leading to two independent LEED $I-V$ structure determinations ${ }^{9,10}$ that provide valuable data with which to benchmark the accuracy of our computational approach. Nonetheless, our general understanding of salt surfaces falls far behind that of metal and semiconductor surfaces, with important issues such as the surface energy and step formation energies of $\mathrm{NaCl}(001)$ remaining unclear. Indeed, this absence of insight into clean $\mathrm{NaCl}(001)$ persists despite several recent theoretical studies of atomic and molecular adsorption on $\mathrm{NaCl}(001) .{ }^{11-14}$

As part of our ongoing work into the chemical and physical properties of salt surfaces, ${ }^{15}$ we aim here to elucidate some of the key structural and energetic properties of flat and stepped $\mathrm{NaCl}(001)$ with density functional theory (DFT). The particular interesting and unresolved issues that we intend to shed light on are (i) the surface energy and atomic structure of $\mathrm{NaCl}(001)$; (ii) the step formation energy of the most commonly observed monoatomic (100)-like steps on $\mathrm{NaCl}(001)$; (iii) the relative stabilities of $\mathrm{Na}$ and $\mathrm{Cl}$ terminated (111)-like steps, their stabilities compared to the neutral (100)-like steps, and insight into what conditions of temperature and pressure (if any) such steps will become stable; and (iv) the electronic structure of $\mathrm{Na}$ and $\mathrm{Cl}$ atoms at $\mathrm{NaCl}(001)$ terraces and steps, aiming, in particular, to understand how these atoms differ from atoms inside the bulk of a $\mathrm{NaCl}$ crystal. To address these issues, we employ three different exchange-correlation functionals: the local-density approximation (LDA), the generalized gradient approximation (GGA) of Perdew, Burke, and Ernzerhof (PBE), ${ }^{16}$ and a revised PBE from $\mathrm{Wu}$ and Cohen (PBE-WC). ${ }^{17}$ The latter 
PBE-WC functional is of interest because it has been shown to outperform PBE for the calculation of various properties of solids and slightly improve over PBE for the surface energy of jellium, but has not yet been applied to compute the surface energy of a real material.

The plan for the remainder of this paper is the following: Section II provides details of the computational setup employed. In Sec. III, bulk $\mathrm{NaCl}$ is discussed, and in Sec. IV, our calculations of flat $\mathrm{NaCl}(001)$ are presented. Section V deals with the structure and energetics of steps at $\mathrm{NaCl}(001)$, and in Sec. VI, several aspects of the electronic structure of flat and stepped $\mathrm{NaCl}(001)$ are addressed. In Sec. VII, we draw some conclusions.

\section{COMPUTATIONAL DETAILS}

All calculations have been performed with DFT within the plane-wave pseudopotential approach, ${ }^{18,19}$ as implemented in the CASTEP code. ${ }^{20}$ Most properties reported here have been computed with the $\mathrm{LDA}^{21}$ and the PBE GGA ${ }^{16}$ exchange-correlation functionals. In addition, the modified PBE-WC functional ${ }^{17}$ has been used for a number of bulk and surface calculations. This functional differs from PBE only in the functional form of the so-called exchange enhancement factor, in the same spirit as the revPBE ${ }^{22}$ and $\mathrm{RPBE}^{23}$ functionals. PBE-WC, as we refer to it here, has been designed to give the exchange hole a more diffuse radial cutoff than the original PBE has, which is believed to be more appropriate for the calculation of solids and their surfaces. ${ }^{17,24}$ Indeed, PBE-WC lives up to this design feature by showing better performance for various properties of solids such as lattice constants, bulk moduli, and the surface energy of jellium. ${ }^{17}$

In the current work, the electron-ion interactions are described with Vanderbilt ultrasoft pseudopotentials ${ }^{25}$ expanded up to a plane-wave cutoff energy of $400 \mathrm{eV}$. LDA pseudopotentials have been used for all LDA calculations, and likewise, GGA (PBE) pseudopotentials have been used for all GGA (PBE and PBE-WC) calculations. Further, we note that the well-known problem of nonlinear core-valence electron exchange and correlation for $\mathrm{Na}$ (Ref. 26) has been eliminated in the present study by treating the $\mathrm{Na} 2 s$ and $2 p$ electrons as valence electrons.

The thicknesses of the $\mathrm{NaCl}$ slabs examined ranged from 2 to 12 layers, and a variety of periodic supercells were used to model the different surface systems considered. For the flat $\mathrm{NaCl}(001)$ surface, a $(1 \times 1)$ unit cell was employed, as is observed in experiment. ${ }^{10}$ For the calculation of monoatomic steps on $\mathrm{NaCl}(001)$, a single layer stripe of $\mathrm{NaCl}(001)$ was placed on top of a four layer $\mathrm{NaCl}(001)$ slab. The stoichiometric "(100)-like" steps were constructed from three atom wide overlayer stripes of $\mathrm{NaCl}(001)$ separated by 1-5 atom wide lower terraces of $\mathrm{NaCl}(001)$ [Fig. 1(a)]. Likewise the polar "(111)-like" steps were constructed from $\mathrm{NaCl}(001)$ overlayer stripes whose "edges" were cut in such a manner as to yield either purely $\mathrm{Cl}$ terminated or purely $\mathrm{Na}$ terminated steps [Fig. 1(b)]. More details of these models will be given below and, in particular, for the (100)-like steps, on which we focus, the dependence of their stability

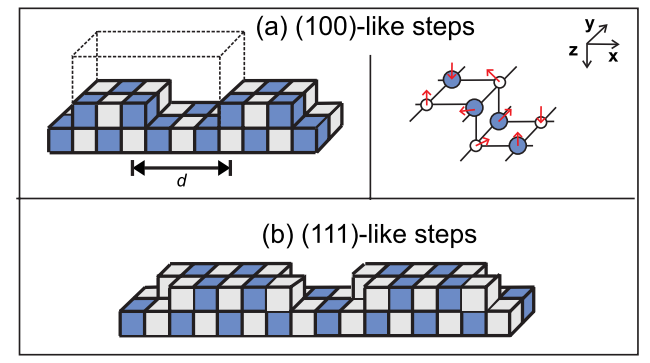

FIG. 1. (Color online) (a) Partial sketch of the model used in the calculation of neutral (100)-like steps on $\mathrm{NaCl}(001)$, comprising a "stripe" of $\mathrm{NaCl}$ atoms on top of the flat $\mathrm{NaCl}(001)$ surface. $d$ is the lower terrace distance between adjacent steps, which is varied in separate calculations to extract the isolated step formation energies of (100)-like steps on $\mathrm{NaCl}(001)$. The dashed rectangular box represents the top half of one specific unit cell used in calculations for the value of $d$ shown. On the right, the direction of the computed displacements of the surface atoms in the vicinity of a step is indicated by the arrows. (b) A partial sketch of the model used in the calculation of the polar (111)-like steps on $\mathrm{NaCl}(001)$. The overlayer stripes are cut to yield either purely $\mathrm{Cl}$ terminated or purely $\mathrm{Na}$ terminated steps. Note that although only one layer of $\mathrm{NaCl}(001)$ is shown, four layer thick $\mathrm{NaCl}$ slabs were used in all step calculations. Dark (blue) cubes or circles represent $\mathrm{Na}$ atoms, whereas light (gray) cubes or circles represent $\mathrm{Cl}$ atoms.

on the width of the upper and lower terraces will be discussed (Sec. V). In every case, a Monkhorst-Pack ${ }^{27} \boldsymbol{k}$-point mesh with the equivalent of at least a $4 \times 4$ sampling within the $1 \times 1$ surface unit cell was used, and the vacuum region between slabs in adjacent cells was in excess of $11 \AA$.

We note that, in addition to the tests of the computational setup discussed in the next section for bulk $\mathrm{NaCl}$, careful tests on the dependence of our computed surface energies [Eq. (4)] and ledge energies [Eq. (5)] with regard to planewave cutoff and $\boldsymbol{k}$-point sampling were performed. These tests, which are reported in Appendix A, reveal that our chosen cutoff energy $(400 \mathrm{eV})$ and $\boldsymbol{k}$-point mesh $(4 \times 4$ sampling within the $1 \times 1$ surface unit cell) yield surface energies and ledge energies converged to within 0.2 and $0.5 \mathrm{meV} / \AA$, respectively, of those obtained with a much higher cutoff $(1200 \mathrm{eV})$ and much denser $\boldsymbol{k}$-point mesh $(16 \times 16$ sampling within the $1 \times 1$ surface unit cell).

\section{BULK NACL}

The properties of bulk $\mathrm{NaCl}$ were examined in order to further establish the accuracy of the current computational setup. In particular, the equilibrium lattice constant, bulk modulus, and various expressions for the energy of bulk $\mathrm{NaCl}$ were computed and compared to experiment and previous theory. ${ }^{28-33}$ These data, which we now discuss, are given in Table I.

Our computed lattice constants with the LDA, PBE, and PBE-WC functionals are 5.48, 5.70, and $5.62 \AA$, respectively, and the computed bulk moduli are 32,23 , and $25 \mathrm{GPa}$. Compared to experiment, PBE overestimates the lattice constant and bulk modulus, whereas LDA underestimates the lattice constant and bulk modulus. This is consis- 
TABLE I. Calculated and measured values for the equilibrium lattice constant $\left(a_{0}\right)$, the bulk modulus $\left(B_{0}\right)$, the formation energy $\left(E_{f}\right)$, the cohesive energy $\left(E^{c o h}\right)$, and the lattice energy (LE) of $\mathrm{NaCl}$. The computed values reported here that come closest to the experimental values for each of the above quantities are indicated in bold.

\begin{tabular}{|c|c|c|c|c|c|c|}
\hline Note & Functional & $\begin{array}{c}a_{0} \\
(\AA)\end{array}$ & $\begin{array}{c}B_{0} \\
(\mathrm{GPa})\end{array}$ & $\begin{array}{c}E_{f} \\
(\mathrm{eV})\end{array}$ & $\begin{array}{l}E^{c o h} \\
(\mathrm{eV})\end{array}$ & $\begin{array}{c}\mathrm{LE} \\
(\mathrm{eV})\end{array}$ \\
\hline \multirow[t]{3}{*}{ This study } & LDA & 5.48 & 32.0 & -3.89 & 6.96 & 8.39 \\
\hline & PBE & 5.70 & 23.2 & -3.63 & 6.16 & 7.59 \\
\hline & PBE-WC & 5.62 & 24.7 & -3.65 & 6.28 & 7.91 \\
\hline \multicolumn{7}{|c|}{ Previous DFT } \\
\hline AE-GTO $^{\mathrm{a}}$ & LDA & 5.48 & 35.2 & & & 8.52 \\
\hline AE-GTO $^{\mathrm{a}}$ & PW91 & 5.75 & 22.9 & & & 7.65 \\
\hline FP-LAPW ${ }^{b}$ & LDA & 5.46 & 32.2 & & & \\
\hline FP-LAPW & PBE & 5.69 & 25.1 & & & \\
\hline PAW-PW & PBE & 5.70 & 23.4 & -3.68 & 6.17 & \\
\hline PP-PW & PBE-WC & 5.62 & 25.4 & & & \\
\hline \multicolumn{7}{|c|}{ Experiment } \\
\hline & & $5.63^{\mathrm{e}}$ & $26.6^{\mathrm{f}}$ & $-4.26^{\mathrm{g}}$ & $6.62^{\mathrm{g}}$ & $8.20^{\mathrm{e}}$ \\
\hline
\end{tabular}

${ }^{\mathrm{a}}$ All electron, full potential, Gaussian-type orbital (Ref. 28).

${ }^{\mathrm{b}}$ All electron, full potential, LAPW (Ref. 29).

${ }^{\mathrm{c}}$ All electron, frozen core PAW, plane wave (Ref. 30).

${ }^{\mathrm{d} P s e u d o p o t e n t i a l,}$ plane wave (Ref. 17).

eReference 31 .

${ }^{\mathrm{f}}$ Reference 32 .

${ }^{g}$ Reference 33.

tent with what is generally known about the performance of these functionals for solids and, for the specific case of $\mathrm{NaCl}$, consistent with previous LDA and PBE calculations. In particular, our values agree well with recent full-potential linearized-augmented plane-wave ${ }^{29}$ (FP-LAPW) and projector augmented wave plus plane-wave ${ }^{30}$ (PAW-PW) studies, as can be seen from Table I. As expected, the PBE-WC functional shows improvements over both LDA and PBE, yielding a lattice constant $(5.62 \AA)$ and a bulk modulus $(25 \mathrm{GPa})$ which are closer to experimental results than the two other functionals, also in agreement with the original PBE-WC paper. $^{17}$

The energetic stability of ionic crystals is typically reported in a variety of different manners, such as the formation energy, the cohesive energy, and the lattice energy. We have computed each of these quantities with the LDA, PBE, and PBE-WC functionals and now discuss each in turn. The formation energy $\left(E_{f}\right)$ of bulk $\mathrm{NaCl}$ at zero Kelvin (neglecting contributions from zero point vibrations) is defined as

$$
E_{f}=E_{\mathrm{NaCl}(\mathrm{s})}-E_{\mathrm{Na}(\mathrm{s})}-\frac{1}{2} E_{\mathrm{Cl}_{2}(\mathrm{~g})},
$$

where $E_{\mathrm{NaCl}(\mathrm{s})}, E_{\mathrm{Na}(\mathrm{s})}$, and $E_{\mathrm{Cl}_{2}(\mathrm{~g})}$ refer to the total energies of bulk $\mathrm{NaCl}$, bulk $\mathrm{Na}$, and gas phase $\mathrm{Cl}_{2}$ molecules, respectively. Our computed formation energies with the LDA, PBE, and PBE-WC functionals are $-3.89,-3.63$, and $-3.65 \mathrm{eV}$, respectively. The corresponding experimental value is $-4.26 \mathrm{eV}$. Thus all functionals employed here pre- dict too small a value of the formation energy. This underestimation is consistent with the only other GGA-PBE report of the formation energy of $\mathrm{NaCl}$, which gave $-3.68 \mathrm{eV} .{ }^{30}$ We have tried to identify the source of the error in the DFT formation energies. It does not appear to come from the $\mathrm{Na}$ bulk reference state since the cohesive energy of bulk $\mathrm{Na}$ is rather accurately computed with all three functionals: 1.25 , 1.08 , and $1.10 \mathrm{eV}$, within the current computational setup for the LDA, PBE, and PBE-WC functionals compared to the experimental value of $1.11 \mathrm{eV}$. A more likely source for the too low $E_{f}$ is an error in the $\mathrm{Cl}_{2}$ molecule, the bond strength of which is overestimated by all three functionals: 3.69, 2.91, and $3.13 \mathrm{eV}$ for the LDA, PBE, and PBE-WC functionals compared to an experimental value of $2.52 \mathrm{eV}$. However, we caution that this is only part of the story and that errors most certainly exist in the $\mathrm{NaCl}$ bulk total energies too, since the $\mathrm{Cl}_{2}$ bond strengths alone do not provide a quantitative account of the computed $E_{f}$ for all three functionals.

The cohesive energy ( $\left.E^{\mathrm{coh}}\right)$ and lattice energy (LE) of $\mathrm{NaCl}$ at zero Kelvin (without corrections for zero point motion) are defined as follows:

$$
\begin{gathered}
E^{\mathrm{coh}}=E_{\mathrm{Na}(\text { atom })}+E_{\mathrm{Cl}(\text { atom })}-E_{\mathrm{NaCl}(\mathrm{s})}, \\
\mathrm{LE}=E_{\mathrm{NaCl}(\mathrm{s})}-E_{\mathrm{Na}^{+}(\text {ion })}-E_{\mathrm{Cl}^{-}(\text {ion })},
\end{gathered}
$$

where $E_{\mathrm{Na} \text { (atom) }}, E_{\mathrm{Na}^{+} \text {(ion) }}, E_{\mathrm{Cl} \text { (atom) }}$, and $E_{\mathrm{Cl}^{-} \text {(ion) }}$ refer to the total energy of isolated $\mathrm{Na}$ and $\mathrm{Cl}$ atoms and ions, respectively. The two quantities differ in how the isolated $\mathrm{Na}$ and 
TABLE II. The relaxation of the interlayer distance $(\Delta)$ as a percentage of the lattice constant and intralayer buckling $\left(\Delta_{i(\mathrm{Cl}-\mathrm{Na})}\right)$ in Angstom as obtained from DFT calculations with 12 layer $\mathrm{NaCl}$ slabs and from LEED $I-V$ analyses. The subscripts designate the various $\mathrm{NaCl}$ layers with 1,2 , and 3 being the top layer, the first subsurface, and second subsurface layers, respectively.

\begin{tabular}{lccccc}
\hline \hline & $\begin{array}{c}\Delta_{12} \\
(\%)\end{array}$ & $\begin{array}{c}\Delta_{23} \\
(\%)\end{array}$ & $\begin{array}{c}\Delta_{1(\mathrm{Cl}-\mathrm{Na})} \\
(\AA)\end{array}$ & $\begin{array}{c}\Delta_{2(\mathrm{Cl}-\mathrm{Na})} \\
(\AA)\end{array}$ & $\begin{array}{c}\Delta_{3(\mathrm{Cl}-\mathrm{Na})} \\
(\AA)\end{array}$ \\
\hline $\mathrm{LDA}$ & -0.55 & 0.07 & 0.072 & -0.009 & 0.000 \\
$\mathrm{PBE}$ & -0.60 & 0.02 & 0.110 & -0.030 & 0.000 \\
$\mathrm{PBE}-\mathrm{WC}$ & -0.46 & 0.10 & 0.107 & -0.035 & 0.000 \\
$\mathrm{NaCl}(001)^{\mathrm{a}}$ & -1.43 & 0.10 & $0.14 \pm 0.003$ & $-0.02 \pm 0.004$ & $0.00 \pm 0.003$ \\
$\mathrm{NaCl} / \mathrm{Pd}(100)^{\mathrm{b}}$ & -3.19 & 0.35 & $0.12 \pm 0.03$ & $0.003 \pm 0.03$ & \\
$\mathrm{NaCl} / \mathrm{Pt}(111)^{\mathrm{b}}$ & -2.84 & 0.35 & $0.12 \pm 0.04$ & $0.01 \pm 0.04$ & \\
\hline
\end{tabular}

${ }^{\mathrm{a} R e f e r e n c e} 10$.

${ }^{\mathrm{b}}$ Reference 9 .

$\mathrm{Cl}$ species are defined: for $E^{\mathrm{coh}}$, it is one comprised of $\mathrm{Na}$ and $\mathrm{Cl}$ atoms, whereas for LE it is one comprised of charged ions. From Table I, it can be seen that all functionals do a reasonable job at predicting $E^{\mathrm{coh}}$, coming within $0.5 \mathrm{eV}(7 \%)$ of the experimental value $(6.62 \mathrm{eV})$. Specifically, the computed values are 6.96, 6.16, and $6.28 \mathrm{eV}$ for LDA, PBE, and PBE-WC, respectively. Again, where comparison with previous DFT calculations is possible, our computed (PBE) value is very close $(0.01 \mathrm{eV}$ away) to the previous PAW-PW report. $^{30}$

For the calculation of the lattice energy, similar quality results are obtained with the computed values of LE being 8.39, 7.59, and 7.91 eV with the LDA, PBE, and PBE-WC functionals, respectively, compared to the experimental value of $8.20 \mathrm{eV}$. We note, however, that for the calculation of the charged $\left(\mathrm{Na}^{+}\right.$and $\left.\mathrm{Cl}^{-}\right)$ions in our periodic supercell, it was necessary to apply the correction of Makov and Payne ${ }^{34}$ to obtain reliable ionization energies and electron affinities for $\mathrm{Na}$ and $\mathrm{Cl}$. Our computed values for LE with the LDA $(8.39 \mathrm{eV})$ and PBE $(7.59 \mathrm{eV})$ functionals are close to the values of 8.52 and $7.65 \mathrm{eV}$ reported before for the LDA and PW91 (Ref. 35) functionals in a periodic DFT study of alkali halides with basis sets comprised of Gaussian-type orbitals. ${ }^{28}$

In conclusion, we have established the accuracy of the current computational setup and made comparisons to experiment and previous theory when possible. As reported before, PBE-WC improves over PBE (and LDA) for the calculation of the lattice constant and bulk modulus of $\mathrm{NaCl}$. However, when it comes to the calculation of the energetics of bulk $\mathrm{NaCl}$, it is difficult to see too much improvement with PBE-WC.

\section{NACL(001)}

\section{A. Structure of $\mathrm{NaCl}(001)$}

The $\mathrm{NaCl}(001)$ surface was examined with the LDA, $\mathrm{PBE}$, and PBE-WC functionals. In Table II, our computed DFT values for some key structural parameters of $\mathrm{NaCl}(001)$, obtained from calculations with 12 layer thick slabs, are reported. In particular, the interlayer spacing and intralayer buckling of the $\mathrm{Na}$ and $\mathrm{Cl}$ atoms of the top three layers are given along with the experimental values determined from the two LEED $I-V$ studies. ${ }^{9,10}$ It is encouraging that all three DFT functionals agree well with each other. Specifically, a contraction of the interlayer distance between the first and second layers $\left(\Delta_{12}\right)$ of about $0.5 \%$ is predicted by all three functionals and a small buckling in the height of the $\mathrm{Na}$ and $\mathrm{Cl}$ atoms in the top layer of $0.07-0.11 \AA$ is obtained. We note that a positive value for the buckling implies that the $\mathrm{Cl}$ atoms have relaxed outward (toward the vacuum) relative to the $\mathrm{Na}$ atoms. The trends in the buckling and relaxations predicted here agree with the LEED analyses, although our predicted first to second layer relaxation at $0.5 \%$ is smaller than that obtained from LEED $(1.4 \%$ from the single crystal study ${ }^{10}$ and $\sim 3 \%$ from the experiments on the thin metal-supported $\mathrm{NaCl}$ films ${ }^{9}$ ). Aside from the relaxation and buckling of the top layer atoms, the atoms in other layers remain almost exactly at their bulk truncated positions. Specifically, the interlayer spacing between the second and third layers $\left(\Delta_{23}\right)$ differs from the bulk value by $\leqslant 0.1 \%$ in our calculations and $0.11 \%$ in experiment, ${ }^{10}$ and the buckling of the $\mathrm{Na}$ and $\mathrm{Cl}$ atoms in the second layer $\left(\Delta_{2(\mathrm{Cl}-\mathrm{Na})}\right)$ is reduced to between -0.01 and $-0.04 \AA$ in our calculations and $-0.02 \AA$ in experiment. ${ }^{10}$ By the time the third layer is reached, both DFT and LEED agree that the bulk, nonbuckled, limit has been reached.

\section{B. Surface energy of $\mathrm{NaCl}(001)$}

The surface energy is a basic thermodynamic quantity of a surface, of crucial importance to the equilibrium shape of crystals. However, it is an incredibly difficult quantity to determine experimentally, ${ }^{36,37}$ and, indeed, most experimental surface energy measurements of $\mathrm{NaCl}$ (and other materials) go back to the $1960 \mathrm{~s}$ and $1970 \mathrm{~s} .{ }^{38}$ For $\mathrm{NaCl}$, this has led to a large range of $11-24 \mathrm{meV} / \AA^{2}$ for the surface energy of $\mathrm{NaCl}(001)$. We illustrate this with the plot in Fig. 2(a) and aim here to make a contribution from first principles theory toward narrowing this range somewhat.

The surface energy is defined as

$$
\gamma=\left(E_{\text {slab }}-N E_{\text {bulk }}\right) / A,
$$

where $E_{\text {slab }}$ is the total energy of the $\mathrm{NaCl}$ slab and $E_{\text {bulk }}$ is the total energy of a $\mathrm{NaCl}$ formula unit in bulk $\mathrm{NaCl}$. The 
(a)

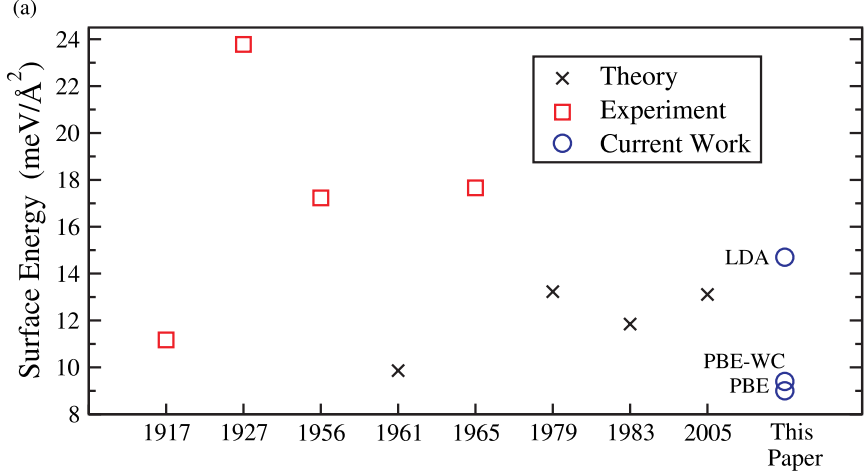

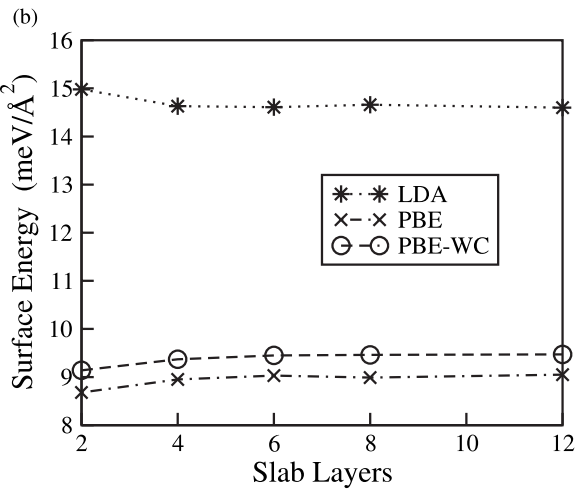

FIG. 2. (Color online) (a) Measured and computed values of the surface energy of $\mathrm{NaCl}(001)$ since 1917, along with the LDA, PBE, and PBE-WC values determined here. The references corresponding to the previously reported values are given in Ref. 38. (b) The dependence of the computed surface energy of $\mathrm{NaCl}(001)$ on the number of $\mathrm{NaCl}$ layers and exchange-correlation functional used.

number of $\mathrm{NaCl}$ formula units in the slab is $N$, and $A$ is the surface area of the slab. For all three exchange-correlation functionals, slabs of 2-12 layer thickness were examined, leading to the computed values of $\gamma$ displayed in Fig. 2(b). Two features of Fig. 2(b) are apparent. First, $\gamma$ is extremely insensitive to the number of layers used in the calculation. The computed values of $\gamma$ with the two layer slabs differ from those computed with the 12 layer slabs, by $<0.5 \mathrm{meV} / \AA^{2}$ for all three functionals. Second, the choice of exchange-correlation functional has a noticeable and significant impact on $\gamma$, with $\gamma$ being $\sim 14.6, \sim 9.0$, and $\sim 9.4 \mathrm{meV} / \AA^{2}$ with the LDA, PBE, and PBE-WC functionals, respectively.

In the absence of reliable experimental measurements of the surface energy of $\mathrm{NaCl}(001)$, it is difficult to say which functional yields the most reliable value of $\gamma$. However, we can make a qualitative judgment by drawing upon experience from other materials. When comparison to experiment is possible, as it most often is for metals, it is typically found that the PBE values are smaller than the experimental ones, with LDA being the closer of the two to experiment. ${ }^{39-41}$ In addition, the recent DFT and diffusion quantum Monte $\mathrm{Carlo}^{42}$ (DMC) studies for the surface energy of $\mathrm{MgO}(001)$ find that the LDA value is about $30 \%$ larger than the PBE value and, moreover, that the LDA value is considerably closer to the, in principle, more reliable DMC result. Finally, we note that the surface energy of $\mathrm{NaCl}(001)$ predicted from the PBE-WC functional is about $4 \%$ larger than the one obtained with PBE. Thus for the surface energy of $\mathrm{NaCl}(001)$, PBE-WC does not appear to offer any significantly improved performance over PBE. Overall, in the absence of a welldefined experimental value or calculation results superior to those provided by DFT, it seems reasonable to suggest that the best estimate of the surface energy of $\mathrm{NaCl}(001)$ is the one proposed here of $9.0-15.0 \mathrm{meV} / \AA^{2}$.

\section{STEPS ON NACL(001)}

Steps are ubiquitous at surfaces and play an interesting and important role in determining the physical and chemical properties of materials. For example, on $\mathrm{NaCl}(001)$ nonpolar monoatomic steps observed with atomic force microscopy have been seen to be the active sites for water adsorption and $\mathrm{NaCl}$ dissolution. ${ }^{4}$ However, quantitative information from either experiment or first principles theory for steps on $\mathrm{NaCl}$ is absent, and in particular, the energetic cost to create steps on $\mathrm{NaCl}$ is not known. Here, we aim to make a start at remedying this situation by determining the formation energies of and relative stabilities between stoichiometric (100)like and nonstoichiometric (111)-like steps.

\section{A. Nonpolar (100)-like steps}

To begin we introduce a quantity, the ledge energy, which is defined as

$$
E_{\text {ledge }}=\left(E_{\text {slab }}-N E_{\text {bulk }}-\gamma A\right) / 2 L,
$$

where $E_{\text {slab }}$ is the total energy of the relaxed stepped surface, which contains $N$ formula units of $\mathrm{NaCl}$. As before, $E_{\text {bulk }}$ is the bulk energy of a $\mathrm{NaCl}$ formula unit and $\gamma$ is the computed $\mathrm{NaCl}(001)$ surface energy. The surface area of the slab is $A$, and $L$ is the length of the step in the unit cell. Since there are two equivalent step edges in each unit cell, the ledge energy is divided by 2 . From the above definition, it can be seen that the ledge energy is the excess energy the stepped surface has over the flat $\mathrm{NaCl}(001)$ surface. This differs from the step formation energy of an isolated step by the step-step interaction between neighboring steps. By examining the dependence of the ledge energy on the width of the terrace that separates neighboring steps, however, the isolated step formation energy $\left(E_{\text {step }}\right)$ can be extracted with the aid of the following relation: ${ }^{43-45}$

$$
E_{\text {ledge }}=E_{\text {step }}+B / d^{2}
$$

where $B / d^{2}$ represents the step-step interaction between neighboring steps and $d$ is the lower terrace length, as shown in Fig. 1. The $1 / d^{2}$ dependence comes from the fact that both elastic and electrostatic effects give rise to a dipolar $\left(1 / d^{2}\right)$ step-step interaction. ${ }^{44,45}$

To obtain the step energy of (100)-like steps, DFT PBE calculations with three different values of the lower terrace width $d$ were performed. Specifically, calculations with $d$ equal to 1,2 , and $3 \mathrm{Na}-\mathrm{Cl}-\mathrm{Na}$ nearest neighbor distances were performed. With the DFT PBE Na-Cl-Na distance of 


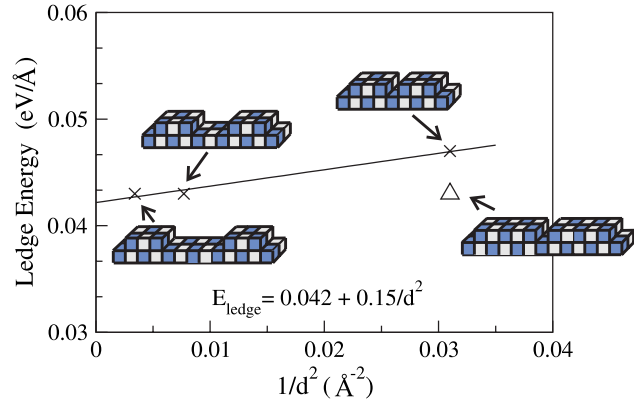

FIG. 3. (Color online) DFT-PBE ledge energy, as defined in Eq. (5), associated with a (100)-like step on $\mathrm{NaCl}(001)$ as a function of the inverse distance between neighboring steps. $d$ is varied from one to three $\mathrm{Na}-\mathrm{Cl}-\mathrm{Na}$ distances, as indicated by the schematic structures. A linear fit [Eq. (6), solid line] to the three points yields a PBE based estimate of the isolated step formation energy $\sim 42 \mathrm{meV} / \AA$. A single point (triangle) obtained with a wider upper terrace (two Na-Cl-Na distances wide) indicates that the computed ledge energy is reasonably insensitive to the width of the upper terrace.

$5.70 \AA$, the width of the lower terrace thus ranges from 5.70 to $17.10 \AA$. The ledge energies obtained from these calculations are plotted in Fig. 3 as a function of $1 / d^{2}$, and from this, a PBE step formation energy of $\sim 42 \mathrm{meV} / \AA$ for monoatomic (100)-like steps on $\mathrm{NaCl}(001)$ is determined.

An additional feature of the data displayed in Fig. 3 is that the interaction between adjacent steps is quite weak since the slope of the fitted line is rather shallow. Indeed, even for the system with the shortest intervening terrace between the steps, i.e., $d=5.70 \AA$, the ledge energy is within $5 \mathrm{meV} / \AA$ of the extrapolated value. For the systems with the two larger intervening terraces the step-step interaction across the lower terrace is essentially zero. ${ }^{46}$ An implication of this is that for this system, although certainly not in general, $E_{\text {ledge }}$ is an excellent approximation for $E_{\text {step }}$.

The data fitted to Eq. (6) and used to extract $E_{\text {step }}$ in Fig. 3 were all obtained with a fixed upper terrace width of one $\mathrm{Na}-\mathrm{Cl}-\mathrm{Na}$ distance. A calculation of the ledge energy with a wider upper terrace, equal to two $\mathrm{Na}-\mathrm{Cl}-\mathrm{Na}$ distances, yields a value of $E_{\text {ledge }}$ that differs from that obtained with the regular upper terrace by only $4 \mathrm{meV} / \AA$. Such a small change demonstrates that $E_{\text {ledge }}$ is also rather insensitive to the upper terrace width and indicates that our computed PBE step formation energy of $\sim 42 \mathrm{meV} / \AA$ is reasonably robust to changes in the surface model employed. For the exchangecorrelation functional, however, it is a different story and, as we saw with the surface energy, the choice of exchangecorrelation functional has a significant impact on the computed value. Specifically, the LDA ledge energy for a stepped surface with a lower terrace width of one $\mathrm{Na}-\mathrm{Cl}-\mathrm{Na}$ distance is $58 \mathrm{meV} / \AA$, compared to the equivalent PBE result of $47 \mathrm{meV} / \AA$. Thus, as with the surface energy, the LDA value exceeds that of the PBE by $\sim 25 \%$. Again, in the absence of experimental measurements for the step formation energy on $\mathrm{NaCl}(001)$, we speculate that the real value of $E_{\text {step }}$ is within the range provided by the $\mathrm{PBE}$ and LDA functionals (40-60 meV/ $\AA$ ).

Before moving to the (111)-like steps, we briefly discuss the optimized structure of the (100)-like steps computed

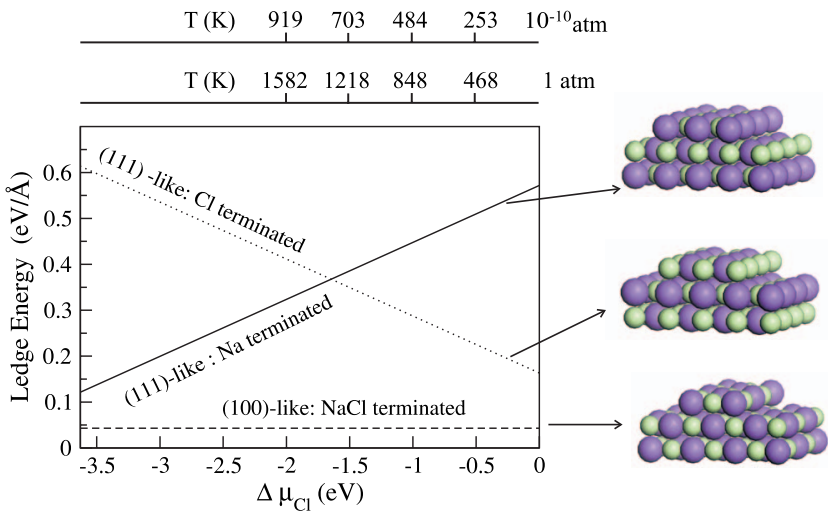

FIG. 4. (Color online) DFT-PBE ledge energy of (100)-like and (111)-like steps as a function of $\mathrm{Cl}_{2}$ chemical potential. The zero of the $\mathrm{Cl}_{2}$ chemical potential axis corresponds to the total energy of gas phase $\mathrm{Cl}_{2}$ molecules, as defined in Eq. (B3). Two temperature scales at 1 and $10^{-10}$ atm are also given as well as partial illustrations of the models used for each type of step calculation. Note that although only two layers of $\mathrm{NaCl}(001)$ are shown, four layer thick $\mathrm{NaCl}$ slabs were used in all step calculations.

here. How the atoms displace in the vicinity of the (100)-like step from their bulk truncated positions is indicated by the arrows on the right of Fig. 1. Overall we find that, as with the flat $\mathrm{NaCl}(001)$ surface, the atoms near the steps only undergo moderate displacements; displacements larger than $0.20 \AA$ from their bulk truncated positions are not observed for any of the atoms in the step simulations. In particular, we find that the atoms not directly located at the step behave essentially as they do on the flat $\mathrm{NaCl}(001)$ surface; i.e., the surface layer $\mathrm{Cl}$ atoms are displaced toward the vacuum, whereas the surface layer $\mathrm{Na}$ atoms move toward the bulk. The $\mathrm{Na}$ and $\mathrm{Cl}$ atoms directly located at the top of the step undergo similar upward and downward movements, but now also undergo displacements inward toward the step. The net result is a $0.13 \AA$ displacement of the upper step $\mathrm{Cl}$ atoms along a plane tilted by $45^{\circ}$ from the surface plane and a $0.18 \AA$ displacement for the upper step $\mathrm{Na}$ atoms along a plane tilted by $27^{\circ}$ from the surface plane. The atoms directly beneath the upper step atoms are naturally affected by the movement of their upper neighbors, and both types of atom undergo an upward relaxation of $0.15 \AA$.

\section{B. Polar (111)-like steps}

Based on the equilibrium crystal shape of $\mathrm{NaCl}$ crystals, it is known that stoichiometric (100)-like steps are more stable than other candidates such as nonstoichiometric (111)-like steps. However, the relative stabilities of (100) and other terminations of step are not known nor is it known under what conditions, if any, other types of step will become favorable. To explore this issue, we have compared the stabilities of (100)-like steps with two alternative polar steps; specifically, a purely $\mathrm{Cl}$ terminated (111)-like step and a purely $\mathrm{Na}$ terminated (111)-like step, illustrations of which are given in Fig. 4. Since the (111)-like terminations are nonstoichiometric, i.e., they contain a different number of $\mathrm{Na}$ and $\mathrm{Cl}$ atoms in the simulation cell, we are unable to directly 

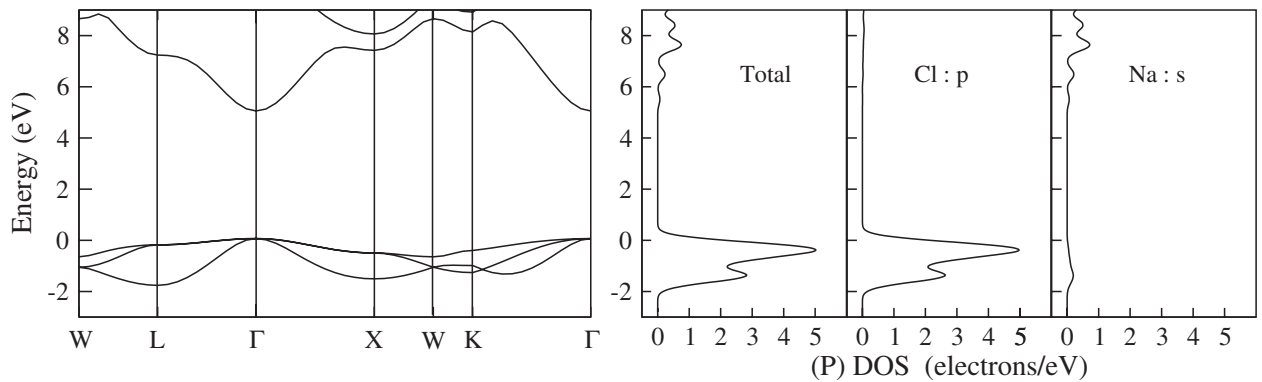

FIG. 5. (a) Band structure of bulk NaCl, as obtained from a DFT-PBE calculation. (b) Total density of states (DOS) and projected densities of states (PDOS), projected onto $\mathrm{Cl} p$ and $\mathrm{Na} s$ states in bulk $\mathrm{NaCl}$. Only the energy interval around the top of the valence band and bottom of the conduction band is shown. A smearing of $0.2 \mathrm{eV}$ has been applied to all DOS and PDOS. The energy zero is the top of the valence band.

determine the ledge energy from Eq. (5), as we did for the (100)-like step. However, it is reasonably straightforward to reformulate the ledge energy as defined in Eq. (5) into a thermodynamic expression based on chemical potentials. This has been done in Appendix B and leads to the following expression for the ledge energy:

$$
\begin{aligned}
E_{\text {ledge }}= & \left\{E_{\text {slab }}-N_{\mathrm{Na}}\left[E_{\mathrm{Na}(\mathrm{s})}+\left(E_{f}-\frac{1}{2} \mu_{\mathrm{Cl}_{2}}\right)\right]\right. \\
& \left.-\frac{1}{2} N_{\mathrm{Cl}}\left(E_{\mathrm{Cl}_{2}(\mathrm{~g})}+\mu_{\mathrm{Cl}_{2}}\right)-\gamma A\right\} / 2 L,
\end{aligned}
$$

where $N_{\mathrm{Na}}$ and $N_{\mathrm{Cl}}$ are the numbers of $\mathrm{Na}$ and $\mathrm{Cl}$ atoms in the simulation cell. The only other new quantity introduced in Eq. (7) is $\mu_{\mathrm{Cl}_{2}}$, the chemical potential of $\mathrm{Cl}_{2}$ molecules in the gas phase. Thus we have an expression for the ledge energy, dependent solely on the chemical potential of $\mathrm{Cl}_{2}$ molecules, which allows us to compare the relative stability of stoichiometric and nonstoichiometric steps. Further, as shown in Appendix B, an estimate of the temperature and pressure dependences of the relative stabilities of differently terminated steps can be obtained by expressing $\mu_{\mathrm{Cl}_{2}}$ in terms of temperature and pressure of the $\mathrm{Cl}_{2}$ gas phase.

The change of ledge energy of the purely $\mathrm{Cl}$ or purely $\mathrm{Na}$ terminated (111)-like steps as well as the stoichiometric (100)-like steps is plotted as a function of $\mu_{\mathrm{Cl}_{2}}$ in Fig. 4. In addition, the temperature dependence of the ledge energy at two specific pressures $[1 \mathrm{~atm}$ and ultrahigh vacuum $\left.\left(10^{-10} \mathrm{~atm}\right)\right]$ is also given. The two key conclusions we draw from Fig. 4 are the following: (i) the (100)-like step is significantly more stable than either termination of (111)-like step at all allowed values of the $\mathrm{Cl}_{2}$ chemical potential. Typically, the ledge energy for the (100)-like step is at least $0.1 \mathrm{eV} / \AA$ lower than either of the nonstoichiometric steps, and (ii) the (111)-like $\mathrm{Cl}$ terminated step is more stable than the $\mathrm{Na}$ terminated step at all values of the $\mathrm{Cl}_{2}$ chemical potential down to $-1.7 \mathrm{eV}$. From the temperature and pressure scales given, one can thus see that the $\mathrm{Cl}$ terminated step is more stable than its $\mathrm{Na}$ counterpart at all experimentally accessible temperatures and pressures.

\section{ELECTRONIC STRUCTURE}

Before ending, we briefly address some aspects of the electronic structure of the $\mathrm{NaCl}$ surfaces examined here. In particular, we discuss how the electronic structures of the atoms at the clean and stepped surfaces differ from the atoms in bulk $\mathrm{NaCl}$.

The density of states (DOS) and projected density of states (PDOS) for bulk NaCl, as obtained with the PBE functional, are plotted in Fig. $5 . \mathrm{NaCl}$ is an insulator with a wide band gap. The computed PBE (direct) Kohn-Sham band gap within the current computational setup is $5.0 \mathrm{eV}$. As expected with such a semilocal GGA functional, this is significantly smaller than the experimental optical value of $8.5 \mathrm{eV}{ }^{47}$ The computed width of the valence band is, however, at $1.6 \mathrm{eV}$, in better agreement with the experimental value of $1.8 \mathrm{eV}{ }^{48}$ The $\mathrm{NaCl}$ valence band is essentially comprised of $\mathrm{Cl} 3 p$ states, as can be seen from the PDOS plot in Fig. 5(b). From the PDOS plot, it can further be seen that the bottom of the conduction band is predicted to be of $\mathrm{Na}(3 s)$ character. This is the conventional interpretation of the $\mathrm{NaCl}$ conduction band. However, there has been some discussion, which is indeed born out by closer inspection of the individual Kohn-Sham orbitals of the bulk $\mathrm{NaCl}$ electronic structure, that the $\mathrm{Cl} 4 \mathrm{~s}$ contribution to the bottom of the valence band is not insignificant. ${ }^{49-51}$

Moving to the surface, we plot the surface band structure and layer resolved DOS in Fig. 6, both of which were obtained from a 12 layer $\mathrm{NaCl}$ slab calculation. The computed valence bandwidth $(1.6 \mathrm{eV})$ and band gap $(5.0 \mathrm{eV})$ are essentially identical to the values computed for bulk $\mathrm{NaCl}$. Further, as predicted long ago by Levine and Mark, ${ }^{52}$ we find that the $\mathrm{NaCl}$ surface does not exhibit any surface states. Indeed, as could be partly anticipated from the small relaxation and buckling at the surface, we do not see any major changes in the electronic structure upon moving from the bulk of $\mathrm{NaCl}$ to the (001) surface.

By looking closely at the layer resolved DOS [Fig. 6(b)], a pronounced but still small effect is visible. Specifically, we see that as we move from the interior of the slab to the surface, the valence band narrows and its center of gravity moves closer to the valence band maximum: compare the DOS for layers 6, 3, 2, and 1 in Fig. 6(b). It is clear from Fig. 6(b), however, that, as with the structural relaxations at the $\mathrm{NaCl}(001)$ surface, the bulk properties are recovered almost immediately with the second layer DOS already very close to that of the bulk. A similar effect is also seen through the $\mathrm{Cl}$ PDOS plots displayed in Fig. 7. Here, the PDOSs associated with a $\mathrm{Cl}$ atom in bulk $\mathrm{NaCl}$, at the surface of $\mathrm{NaCl}(001)$, 
(a)

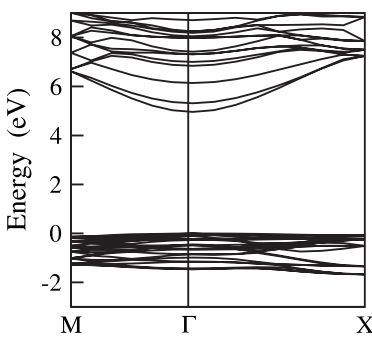

(b)

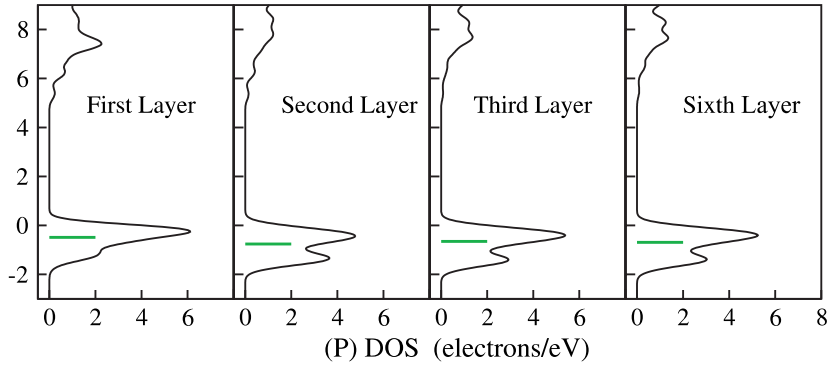

FIG. 6. (Color online) (a) Band structure of a 12 layer $\mathrm{NaCl}(001)$ slab, as obtained from a DFT-PBE calculation. (b) The layer resolved density of states of the first three layers and the sixth layer of a 12 layer $\mathrm{NaCl}(001)$ slab. The horizontal (green) line indicates the center of the valence band in each case. The energy zero is the top of the valence band.

and at a step on $\mathrm{NaCl}(001)$ are displayed. Again, one can see that the $\mathrm{Cl} 3 p$ valence band center moves closer to the valence band maximum as one goes from a $\mathrm{Cl}$ atom in bulk to one at a step. Correlated with these changes in the DOS is an increase in the computed Mulliken charge of the $\mathrm{Na}$ and $\mathrm{Cl}$ atoms upon moving from the bulk to the surface, to the steps, as can be seen from Table III.

\section{CONCLUSIONS}

To recap, bulk $\mathrm{NaCl}$ and flat and stepped $\mathrm{NaCl}(001)$ have been examined with density functional theory. The properties of bulk $\mathrm{NaCl}$ have been computed with the LDA, PBE, and PBE-WC exchange-correlation functionals. Our results agree with previous theoretical studies, and the PBE-WC functional offers improved performance over both LDA and PBE in the description of key bulk properties such as the equilibrium lattice constant and bulk modulus. However, when it comes to the calculation of energetic properties of bulk $\mathrm{NaCl}$ such as the formation energy, cohesive energy, and lattice energy, it is difficult to see too much improvement with PBE-WC compared to PBE.

For the structure of $\mathrm{NaCl}(001)$, we find only a small inward relaxation of $\sim 0.6 \%$ for the top layer and a considerably smaller relaxation for the second to third interlayer distances $(\sim 0.06 \%)$. A small buckling between $\mathrm{Cl}$ and $\mathrm{Na}$

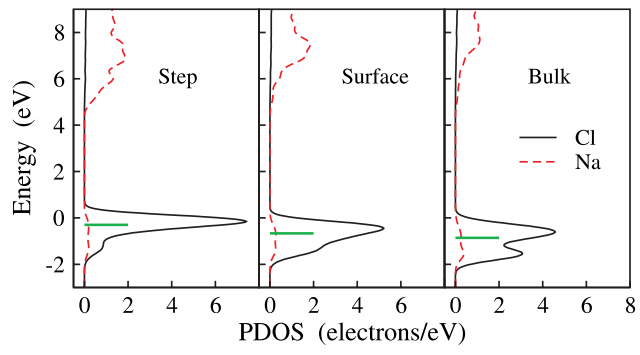

FIG. 7. (Color online) PDOS of a single $\mathrm{Cl}$ atom (solid line) and a single $\mathrm{Na}$ atom (dashed line) positioned at the step, the surface, and the interior (bulk) of a stepped $\mathrm{NaCl}(001)$ slab, as obtained from a DFT-PBE calculation. Only the top of the valence band $(\mathrm{Cl} 3 p)$ and the bottom of the conduction band are displayed. The horizontal (green) line indicates the position of the center of the valence band in each case. The energy zero is the top of the valence band. atoms in the top layer, $\sim 0.1 \AA$, is also predicted. All three exchange-correlation functionals agree on this behavior, which is largely in agreement with the LEED $I-V$ analyses, ${ }^{9,10}$ although the absolute magnitude of the top layer relaxation is somewhat smaller than the values of $1.4 \%$ and $\sim 3 \%$ that come from LEED.

The surface energy of $\mathrm{NaCl}(001)$ is estimated to be in the range from 9 to $15 \mathrm{meV} / \AA^{2}$, as obtained from the three exchange-correlation functionals. In light of the absence of reliable experimental surface energy measurements of $\mathrm{NaCl}(001)$, it is difficult to identify one functional as being clearly superior to the others. However, we have discussed how the surface energies obtained from the LDA and PBE functionals often straddle the true value, with LDA likely to be the closer of the two. An improved estimate of the surface energy of $\mathrm{NaCl}(001)$ must await either new experiments or more potent calculations than those provided in the current work, both of which are now clearly needed.

The energetics of steps on $\mathrm{NaCl}(001)$ has been examined. The formation energy of isolated (100)-like steps on $\mathrm{NaCl}(100)$ is $\sim 42 \mathrm{meV} / \AA$ as obtained from PBE, with the LDA value expected to be about $20 \%$ larger. Placing our computed step energy in a broader context, we note that it is a rather small step formation energy, close to the noble metals silver and copper, for example. ${ }^{44,45,53}$ Further, the interaction between neighboring steps is small, never more than $5 \mathrm{meV} / \AA$ for the systems examined here.

The ledge energy of polar (111)-like steps has also been evaluated, allowing us to compare the relative stabilities of purely $\mathrm{Cl}$ or purely $\mathrm{Na}$ terminated steps with each other and with the neutral (100)-like steps. It is found that the (100)like terminated steps are significantly more stable than either of the (111)-like terminated steps at all allowed values of the chlorine chemical potential. Further, it is found that the $\mathrm{Cl}$

TABLE III. The computed DFT-PBE Mulliken charges on $\mathrm{Cl}$ and $\mathrm{Na}$ atoms in bulk $\mathrm{NaCl}$, at the (001) surface of $\mathrm{NaCl}$, and at the stoichiometric (100)-like steps.

\begin{tabular}{lcc}
\hline \hline Charge $(e)$ & $\mathrm{Na}$ & $\mathrm{Cl}$ \\
\hline Bulk & 0.54 & -0.54 \\
Surface & 0.61 & -0.59 \\
Step & 0.67 & -0.62 \\
\hline \hline
\end{tabular}


(a)

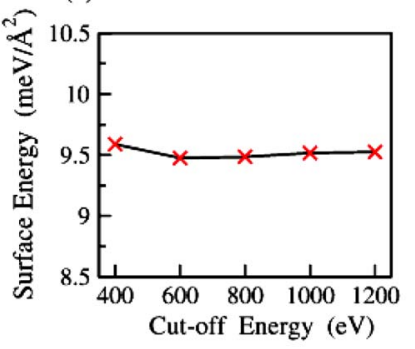

(c)

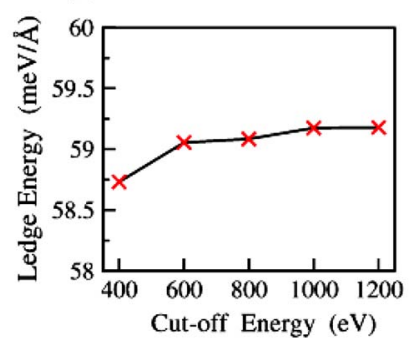

(b)

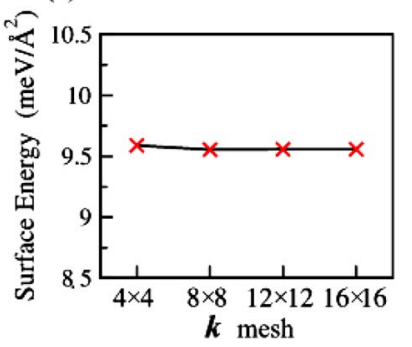

(d)

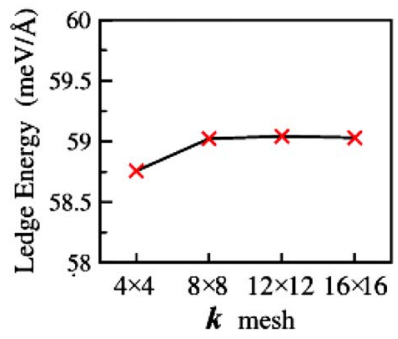

FIG. 8. (Color online) Variation of the computed PBE surface energy of a four layer $\mathrm{NaCl}(001)$ slab with (a) plane-wave cutoff energy and (b) $\boldsymbol{k}$-point mesh. [(c) and (d)] As for (a) and (b) but now for the ledge energy.

terminated steps are more stable than the Na terminated steps down to values of $\mu_{\mathrm{Cl}}=-1.7 \mathrm{eV}$, which effectively means that $\mathrm{Cl}$ terminated steps are more stable than $\mathrm{Na}$ terminated steps at all accessible temperatures and pressures.

Finally, the electronic structure of bulk $\mathrm{NaCl}$, flat $\mathrm{NaCl}(001)$, and stepped $\mathrm{NaCl}(001)$ has been briefly discussed. As with the atomic structure, the electron density in the surface region is not perturbed to any great extent by the presence of the surface. The main effect is a small narrowing and upshift of the valence band DOS as one moves from the bulk to the subsurface region, to the flat surface, and to the steps.

\section{ACKNOWLEDGMENT}

A.M. has been supported through the EURYI award scheme. See www.esf.org/euryi.

\section{APPENDIX A}

Some of the tests carried out in order to determine the numerical accuracy of the computational setup employed in this study are reported here. Specifically, convergence tests on the dependence of the surface energy on the plane-wave cutoff [Fig. 8(a)] and $\boldsymbol{k}$-point mesh [Fig. 8(b)] used are presented as are convergence tests on the dependence of the ledge energy on the plane-wave cutoff [Fig. 8(c)] and $\boldsymbol{k}$-point mesh [Fig. 8(d)]. In all calculations, a fixed four layer $\mathrm{NaCl}$ slab was used and the Monkhorst-Pack $\boldsymbol{k}$-point meshes reported correspond to the sampling per $1 \times 1$ surface unit cell. It can be seen from Fig. 8 that our chosen cutoff energy $(400 \mathrm{eV})$ and $\boldsymbol{k}$-point mesh $(4 \times 4)$ yield surface energies and ledge energies converged to within $0.2 \mathrm{meV} / \AA^{2}$ and $0.5 \mathrm{meV} / \AA$, respectively, of those obtained with a much

higher cutoff $(1200 \mathrm{eV})$ and much denser $\boldsymbol{k}$-point mesh (16 $\times 16 \times 1)$.

\section{APPENDIX B}

Here, we briefly sketch the thermodynamic route that takes us from Eq. (5) to Eq. (7), which enables the ledge energy of stoichiometric and nonstoichiometric steps on $\mathrm{NaCl}(001)$ to be compared. As given in Eq. (5), the ledge is

$$
E_{\text {ledge }}=\left(E_{\text {slab }}-E_{\text {bulk }}-\gamma A\right) / 2 L,
$$

where $E_{\text {bulk }}$ is the energy of the (stoichiometric) $\mathrm{NaCl}$ formula unit in bulk NaCl. The well-known problem is that one cannot define the energy of the atomic species in a compound like $\mathrm{NaCl}$, and thus one cannot determine $E_{\text {ledge }}$ for a nonstoichiometric system, i.e., one in which there are different numbers of $\mathrm{Na}$ and $\mathrm{Cl}$ atoms. However, by employing thermodynamics and considering $\mathrm{Na}$ and $\mathrm{Cl}$ as "subunits" of $\mathrm{NaCl}$, the problem can be quite effectively sidestepped.

To begin, we consider the equilibrium condition for $\mathrm{NaCl}$, which we express as

$$
\left(E_{\mathrm{Na}(\mathrm{s})}+\mu_{\mathrm{Na}}\right)+\frac{1}{2}\left(E_{\mathrm{Cl}_{2}(\mathrm{~g})}+\mu_{\mathrm{Cl}_{2}}\right)=E_{f}+E_{\mathrm{NaCl}(\mathrm{s})},
$$

where, as before, $E_{f}$ is the formation energy of $\mathrm{NaCl}$, and $E_{\mathrm{Na}(\mathrm{s})}, E_{\mathrm{Cl}_{2}(\mathrm{~g})}$, and $E_{\mathrm{NaCl}(\mathrm{s})}$ are the total energies of $\mathrm{Na}$ atoms in bulk $\mathrm{Na}$, gas phase $\mathrm{Cl}_{2}$ molecules, and bulk $\mathrm{NaCl}$, respectively. Setting the total energies to zero yields a formation energy for $\mathrm{NaCl}$ that is now given by the chemical potentials of chlorine molecules, $\mu_{\mathrm{Cl}_{2}}$, and $\mathrm{Na}$ atoms, $\mu_{\mathrm{Na}}$ :

$$
\mu_{\mathrm{Na}}+\frac{1}{2} \mu_{\mathrm{Cl}_{2}}=E_{f}
$$

If we choose the chemical potential of $\mathrm{Cl}_{2}, \mu_{\mathrm{Cl}_{2}}$, as the only independent variable, then its accessible range is obviously given by

$$
E_{f} \leqslant \frac{1}{2} \mu_{\mathrm{Cl}_{2}} \leqslant 0
$$

where the lower limit, the "chlorine poor" limit, is the computed heat of formation of $\mathrm{NaCl}$, which with the PBE functional is $-3.63 \mathrm{eV}$ (Table I). By construction, the other limit, the "chlorine rich" limit, is given by $E_{\mathrm{Cl}_{2}(\mathrm{~g})}$, the computed total energy of gas phase chlorine molecules. Combining Eqs. (B1) and (B2) thus leads to the expression for the ledge energy given in Eq. (7),

$$
\begin{aligned}
E_{\text {ledge }}= & \left\{E_{\text {slab }}-N_{\mathrm{Na}}\left[E_{\mathrm{Na}(\mathrm{s})}+\left(E_{f}-\frac{1}{2} \mu_{\mathrm{Cl}_{2}}\right)\right]\right. \\
& \left.-\frac{1}{2} N_{\mathrm{Cl}}\left(E_{\mathrm{Cl}_{2}(\mathrm{~g})}+\mu_{\mathrm{Cl}_{2}}\right)-\gamma A\right\} / 2 L,
\end{aligned}
$$

where $E_{\text {ledge }}$ now depends only on the chemical potential of $\mathrm{Cl}_{2}$ molecules.

Further, treating the $\mathrm{Cl}_{2}$ molecules as an ideal-gas reservoir allows us to extract the temperature and pressure dependences of the chlorine chemical potential, $\mu_{\mathrm{Cl}}(T, p)$, which 
gives crude temperature and pressure dependences for the relative stabilities of the differently terminated steps on $\mathrm{NaCl}(001)$. Specifically, the chlorine chemical potential is given by

$$
\begin{aligned}
\mu_{\mathrm{Cl}}(T, p) & =\frac{1}{2} E_{\mathrm{Cl}_{2}(\mathrm{~g})}+\Delta \mu_{\mathrm{Cl}}(T, p) \\
& =\frac{1}{2}\left[E_{\mathrm{Cl}_{2}(\mathrm{~g})}+\mu_{\mathrm{Cl}_{2}}\left(T, p^{0}\right)+k_{B} T \ln \left(\frac{p_{\mathrm{Cl}_{2}}}{p^{0}}\right)\right],
\end{aligned}
$$

where, as before, $E_{\mathrm{Cl}_{2}(\mathrm{~g})}$ is the computed DFT total energy of a $\mathrm{Cl}_{2}$ molecule and $\Delta \mu_{\mathrm{Cl}}(T, p)$ thus incorporates the temperature and pressure dependences of the chlorine chemical potential, with the contribution from rotations and vibrations of the molecule, as well as the ideal-gas entropy at $p^{0}=1 \mathrm{~atm}$ incorporated in $\mu_{\mathrm{Cl}_{2}}\left(T, p^{0}\right)$. Boltzmann's constant and temperature are, of course, $k_{B}$ and $T$, respectively. $\mu_{\mathrm{Cl}_{2}}\left(T, p^{0}\right)$ can be computed from first principles or, as we have done here, taken from thermodynamic tables ${ }^{33}$ yielding the chlorine chemical potential at any particular temperature and pressure of interest. *michaeli@fhi-berlin.mpg.de

${ }^{1}$ R. J. MacDonald, E. C. Taglauer, and K. R. Wandelt, Surface Science: Principles and Current Applications (Springer, Berlin, 1996).

${ }^{2}$ K. W. Kolasinski, Surface Science: Foundations of Catalysis and Nanoscience (Wiley, New York, 2002).

${ }^{3}$ P. W. Tasker, Philos. Mag. A 39, 119 (1979).

${ }^{4}$ Q. Dai, J. Hu, and M. Salmeron, J. Phys. Chem. B 101, 1994 (1997); A. Verdaguer, G. M. Sacha, H. Bluhm, and M. Salmeron, Chem. Rev. (Washington, D.C.) 106, 1478 (2006).

${ }^{5}$ R. Bennewitz A. S. Foster, L. N. Kantorovich, M. Bammerlin, C. Loppacher, S. Schar, M. Guggisberg, E. Meyer, and A. L. Shluger, Phys. Rev. B 62, 2074 (2000).

${ }^{6}$ R. Bennewitz, M. Bammerlin, M. Guggisberg, C. Loppacher, A. Barato, E. Meyer, and H.-J. Güntherodt, Surf. Interface Anal. 27, 462 (1999).

${ }^{7}$ C. Barth and C. R. Henry, Phys. Rev. Lett. 98, 136804 (2007).

${ }^{8}$ V. E. Henrich and P. A. Cox, The Surface Science of Metal Oxides (Cambridge University Press, Cambridge, 1996).

${ }^{9}$ J. G. Roberts, S. Hoffer, M. A. Van Hove, and G. A. Somorjai, Surf. Sci. 437, 75 (1999).

${ }^{10}$ J. Vogt and H. Weiss, Surf. Sci. 491, 155 (2001).

${ }^{11}$ J. M. Park, J. H. Cho, and K. S. Kim, Phys. Rev. B 69, 233403 (2004).

${ }^{12}$ Y. Yang, S. Meng, and E. G. Wang, Phys. Rev. B 74, 245409 (2006).

${ }^{13}$ H. Meyer, P. Entel, and J. Hafner, Surf. Sci. 488, 177 (2001).

${ }^{14}$ S. Briques, S. Picaud, C. Girardet, P. N. M. Hoang, J. Heidberg, and A. Voßberg, J. Chem. Phys. 109, 6435 (1998).

${ }^{15}$ B. Li, A. Michaelides, and M. Scheffler, Phys. Rev. Lett. 97, 046802 (2006).

${ }^{16}$ J. P. Perdew, K. Burke, and M. Ernzerhof, Phys. Rev. Lett. 77, 3865 (1996); 78, 1396 (1997).

${ }^{17}$ Z. Wu and R. E. Cohen, Phys. Rev. B 73, 235116 (2006).

${ }^{18}$ M. C. Payne, M. P. Teter, D. C. Allan, T. A. Arias, and J. D. Joannopoulos, Rev. Mod. Phys. 64, 1045 (1992).

${ }^{19}$ M. Bochstedte, A. Kley, J. Neugebauer, and M. Scheffler, Comput. Phys. Commun. 107, 187 (1997).

${ }^{20}$ S. J. Clark, M. D. Segall, C. J. Pickard, P. J. Hasnip, M. I. J. Probert, K. Refson, and M. C. Payne, Z. Kristallogr. 220, 567 (2005); M. D. Segall, P. L. D. Lindan, M. J. Probert, C. J. Pickard, P. J. Hasnip, S. J. Clark, and M. C. Payne, J. Phys.: Condens. Matter 14, 2717 (2002).
${ }^{21}$ J. P. Perdew and A. Zunger, Phys. Rev. B 23, 5048 (1981).

${ }^{22}$ Y. Zhang and W. Yang, Phys. Rev. Lett. 80, 890 (1998).

${ }^{23}$ B. Hammer, L. B. Hansen, and J. K. Nørskov, Phys. Rev. B 59, 7413 (1999).

${ }^{24}$ V. N. Staroverov, G. E. Scuseria, J. Tao, and J. P. Perdew, Phys. Rev. B 69, 075102 (2004).

${ }^{25}$ D. Vanderbilt, Phys. Rev. B 41, 7892 (1990).

${ }^{26}$ S. G. Louie, S. Froyen, and M. L. Cohen, Phys. Rev. B 26, 1738 (1982).

${ }^{27}$ H. J. Monkhorst and J. D. Pack, Phys. Rev. B 13, 5188 (1976).

${ }^{28}$ G. Sandrone and D. A. Dixon, J. Phys. Chem. A 102, 10310 (1998).

${ }^{29}$ M. Chall, B. Winkler, P. Blaha, and K. Schwarz, J. Phys. Chem. B 104, 1191 (2000).

${ }^{30}$ J. Paier, K. Hummer, G. Kresse, I. C. Gerber, and J. G. Ángyán, J. Chem. Phys. 124, 154709 (2006).

${ }^{31}$ Handbook of Chemistry and Physics, 76th ed. (CRC, Boca Raton, FL, 1995).

${ }^{32}$ T. J. Lewis, A. Lehoczky, and C. V. Briscoe, Phys. Rev. 161, 877 (1967).

${ }^{33}$ NIST-JANAF Thermochemical Tables, edited by M. W. Chase, Jr. (AIP, New York, 1998).

${ }^{34}$ G. Makov and M. C. Payne, Phys. Rev. B 51, 4014 (1995).

${ }^{35}$ J. P. Perdew, J. A. Chevary, S. H. Vosko, K. A. Jackson, M. R. Pederson, D. J. Singh, and C. Fiolhais, Phys. Rev. B 46, 6671 (1992).

${ }^{36}$ M. C. Desjonquères and D. Spanjaard, Concepts in Surface Physics (Springer, Amsterdam, 1998).

${ }^{37}$ V. K. Kumikov and K. B. Khokonov, J. Appl. Phys. 54, 1346 (1983).

${ }^{38}$ F. M. Jaegar, Z. Anorg. Chem., 101, 1 (1917); S. G. Lipsett, F. M. G. Johnson, and O. Maass, J. Am. Chem. Soc. 49, 1940 (1927); G. C. Benson, H. P. Schreiber, and F. Vanzeggeren, Can. J. Chem. 34, 1553 (1956); G. C. Benson, J. Chem. Phys. 35, 2113 (1961); P. L. Gunshall and G. E. Gross, J. Appl. Phys. 36, 2459 (1965); P. W. Tasker, Philos. Mag. A, 39, 119 (1979); P. A. Cox, F. W. H. Dean, and A. A. Williams, Vacuum 33, 839 (1983); T. Zykova-Timan, D. Ceresoli, U. Tartaglino, and E. Tosatti, Phys. Rev. Lett. 94, 176105 (2005).

${ }^{39}$ J. L. F. Da Silva, C. Stampfl, and M. Scheffler, Surf. Sci. 600, 703 (2006).

${ }^{40}$ D. K. Yu and M. Scheffler, Phys. Rev. B 70, 155417 (2004).

${ }^{41}$ This apparently superior performance of LDA compared to that of PBE for the surface energy of metals has been rationalized by 
recalling that, due to a favorable cancellation of errors, LDA outperforms PBE for the calculation of the surface energy of jellium, too (Ref. 24). Of course, jellium is not a good model for $\mathrm{NaCl}$, but, nonetheless, the relative magnitude of the LDA and PBE surface energies computed here is similar to what is seen for jellium.

${ }^{42}$ D. Alfè and M. J. Gillan, J. Phys.: Condens. Matter 18, L435 (2006).

${ }^{43}$ V. B. Shenoy and C. V. Ciobanu, Phys. Rev. B 67, 081402(R) (2003).

${ }^{44}$ E. D. Williams, Surf. Sci. 299, 502 (1994).

${ }^{45}$ H. Jeong and E. D. Williams, Surf. Sci. Rep. 34, 171 (1999).

${ }^{46}$ The weak interaction between adjacent steps is further born out by analysis of the atomic and electronic structures of the relaxed (i.e., optimized) steps obtained from our calculations with the different sized terraces. Specifically, the relaxation of the atoms at the steps separated by the smallest intervening terrace $(5.70 \AA)$ differs from those with the largest terrace $(17.10 \AA$ ) by $0.01 \AA$ at most. Likewise the Mulliken charges and partial density of states of the atoms at the steps are essentially identical in the systems with the longer and shorter intervening terraces.

${ }^{47}$ N. O. Lipari and A. B. Kunz, Phys. Rev. B 3, 491 (1971).

${ }^{48}$ G. K. Wertheim, J. E. Rowe, D. N. E. Buchanan, and P. H. Citrin, Phys. Rev. B 51, 13675 (1995).

${ }^{49}$ J. C. Slater, Quantum Theory of Molecules and Solids, 2: Symmetry and Energy Bands in Crystals (McGraw-Hill, New York, 1976).

${ }^{50}$ P. K. de Boer and R. A. de Groot, Phys. Lett. A 256, 227 (1999).

${ }^{51}$ C. Freysoldt, Ph.D Thesis, Freie Universität, Berlin, 2007.

${ }^{52}$ J. D. Levine and P. Mark, Phys. Rev. 144, 751 (1966).

${ }^{53}$ J. Kollar, L. Vitos, B. Johansson, and H. L. Skriver, Phys. Status Solidi B 217, 405 (2000). 